\title{
Effet de Prétraitements sur la Germination des Semences d'Acacia senegal (L.) Willd. (Mimosaceae) dans la Zone Sahélienne du Cameroun
}

\begin{abstract}
Hamawa Yougouda,
Département d'Agriculture, Elevage et Produits Dérivés, Ecole Nationale

Supérieure Polytechnique, Université de Maroua, Cameroun

Faculté des Sciences, Université de Ngaoundéré, Laboratoire de Biodiversité et Développement Durable, Ngaoundéré, Cameroun

Baye-Niwah Claudette,

Département des Sciences de la Vie et de la Terre, Ecole Normale

Supérieure, Université de Maroua, Cameroun

Faculté des Sciences, Université de Ngaoundéré, Laboratoire de Biodiversité et Développement Durable, Ngaoundéré, Cameroun

Kepwa Fils Bill Franck Steve,

Département d'Agriculture, Elevage et Produits Dérivés, Ecole Nationale

Supérieure Polytechnique, Université de Maroua, Cameroun
\end{abstract}

Mapongmetsem Pierre Marie,

Faculté des Sciences, Université de Ngaoundéré, Laboratoire de Biodiversité et Développement Durable, Ngaoundéré, Cameroun

Doi:10.19044/esj.2020.v16n3p263～URL:http://dx.doi.org/10.19044/esj.2020.v16n3p263

Résumé

Le gommier du Sénégal (Acacia senegal (L.) Willd. ; Mimosaceae) est une essence d'une grande importance socio-économique. Il est largement répandu sur l'ensemble des zones tropicales arides et semi-arides d'Afrique. Sa grande plasticité et sa forte résilience à la sécheresse ont fait de lui une espèce indispensable pour de grands reboisements de production comme de protection dans la zone sahélienne du Cameroun. Les semences de cette espèce sont caractérisées généralement par une dormance surtout tégumentaire. Une expérimentation a été menée pour déterminer l'effet de différents prétraitements sur la germination des graines de cette espèce. Les semences ont subi trois prétraitements (scarification à la lame de rasoir, trempage dans l'eau bouillante et traitement à l'acide sulfurique concentré pendant 9 min et rinçage à l'eau du robinet). Les résultats obtenus ont montré que la germination a été meilleure (délai de germination, taux de germination et vitesse de germination) avec la scarification. Les prétraitements à l'acide 
sulfurique concentré et à l'eau bouillante ont des effets non négligeables sur la germination de l'espèce étudiée. La scarification des graines et leur ébouillantage ont donné des taux de germination très élevés (94\% et $70 \%$ respectivement, 16 jours après semis) et ont permis d'accélérer sensiblement la vitesse de germination. Du fait de leur simplicité et de leur faible coût, ces deux techniques peuvent être recommandées aux planteurs pour la réalisation du semis en pépinière.

Mots clés : Acacia Senegal, Prétraitement, Dormance, Vitesse De Germination, Cameroun

\title{
Effect of Pretreatments on the Germination of Acacia senegnal (L.) Willd. seeds (Mimosaceae) in Sahelian zone of Cameroon
}

\section{Hamawa Yougouda,}

Département d'Agriculture, Elevage et Produits Dérivés, Ecole Nationale

Supérieure Polytechnique, Université de Maroua, Cameroun

Faculté des Sciences, Université de Ngaoundéré, Laboratoire de Biodiversité et Développement Durable, Ngaoundéré, Cameroun

\section{Baye-Niwah Claudette,}

Département des Sciences de la Vie et de la Terre, Ecole Normale

Supérieure, Université de Maroua, Cameroun

Faculté des Sciences, Université de Ngaoundéré, Laboratoire de Biodiversité et Développement Durable, Ngaoundéré, Cameroun

\section{Kepwa Fils Bill Franck Steve,}

Département d'Agriculture, Elevage et Produits Dérivés, Ecole Nationale

Supérieure Polytechnique, Université de Maroua, Cameroun

\section{Mapongmetsem Pierre Marie,}

Faculté des Sciences, Université de Ngaoundéré, Laboratoire de Biodiversité et Développement Durable, Ngaoundéré, Cameroun

\begin{abstract}
The gum tree of Senegal (Acacia senegal (L.) Willd., Mimosaceae) is an essence of great socio-economic importance. It is widely distributed throughout Africa's arid and semi-arid tropics. Its great plasticity and its strong resilience to drought have made it an indispensable species for large
\end{abstract}


reforestation of production as of protection in the Sahelian zone of Cameroon. The seeds of this species are generally characterized by a dormancy mostly integumentary. An experiment was conducted to determine the effect of different pretreatments on seed germination of this species. The seeds underwent three pretreatments (razor blade scarification, boiling water soaking and concentrated sulfuric acid treatment for 9 min and rinsing with tap water). The results obtained showed that germination was better (germination time, percentage of germination and germination rate) with scarification. Pretreatments with concentrated sulfuric acid and boiling water have an effect on the germination of the species studied. Seed scarification and the boiling water gave very high germination percentages (94\% and $70 \%$ respectively, 16 days after sowing) and significantly accelerated the germination rate. Because of their simplicity and low cost, both of these techniques can be recommended to farmers for nursery sowing.

Keywords: Acacia Senegal, Pretreatment, Dormancy, Germination Rate, Cameroon

\section{Introduction}

La forte variabilité climatique combinée aux facteurs anthropiques, a sérieusement affecté les grands équilibres écologiques et entraîné une dégradation des ressources naturelles, des sols et des agro-écosystèmes. Cette situation a rendu les systèmes de production agricole vulnérables. Dans ce contexte, des programmes de développement basés sur les plantations d'espèces végétales à croissance rapide, d'intérêts socio-économiques, parfaitement adaptées au climat aride et surtout qui présentent divers avantages pour le développement rural ont été mis sur pied. C'est ainsi que des plantations d'espèces végétales présentant une haute valeur ajoutée économiquement et qui sont écologiquement utiles telles que Azadirachta indica, Anacardium occidental, Vitellaria paradoxa, Acacia senegal ont fait l'objet de plantation dans la zone. Parmi ces espèces végétales, A. senegal connaît un essor considérable car au-delà des avantages environnementaux des plantations d'A. senegal comme la protection des sols contre l'érosion, l'amélioration de la fertilité des sols (Dommergues et al., 1999 ; Eisa et al., 2008 ; Abdou et al., 2013) et la réduction du taux de déforestation, cette plante présente un intérêt économique certain par sa production de gomme.

Acacia senegal (L.) Willd. (Mimosacées), est une espèce largement répandue dans les zones tropicales arides et semi-arides d'Afrique, comprises entre les isohyètes 150 et $600 \mathrm{~mm}$ (Jamal et Huntsinger, 1993). Son aire de distribution s'étend principalement du Sénégal au Soudan (Brenan, 1983 ; Ickowicz et al., 2005). Dans la zone sahélienne du Cameroun, le bois lourd d'A. senegal est préféré en tant que combustible au bois d'autres espèces 
ligneuses. Les feuilles et les gousses sont riches en protéines digestibles et constituent une source importante de fourrage en saison des pluies et au début de la saison sèche (Ickowicz et al., 2005 ; Diallo et al., 2012). Dans certains pays, A. senegal est surtout utilisé pour la revégétalisation et pour la fixation des dunes. Au Soudan, A. senegal est utilisé dans des systèmes agroforestiers, en combinaison avec des cultures maraîchères, des plantes fourragères annuelles et d'autres cultures comme le mil, le niébé (Anderson \& Sinclair, 1993 ; Saïdou et al., 2018).

En zone sud-sahélienne ou soudano-sahélienne du Cameroun, les plantations d'Acacia senegal contribuent à régénérer des sols dégradés par la culture continue (Harmand et al., 1997), sous forme de jachère améliorée, tout en fournissant du bois et du fourrage (Peltier et Eyog, 1988 ; Peltier, 1993). Outre ces utilisations, A. senegal est la principale espèce productrice de gomme. Au Cameroun, la production est estimée à 4411 tonnes en 2014 (CNUCED, 2016). En dépit de son importance socio-économique, de nombreuses difficultés sont rencontrées dans sa multiplication et sa régénération spontanée. En raison de l'action anthropique, les incendies et le surpâturage, ainsi qu'aux caractères intrinsèques à l'espèce (dureté tégumentaire de la graine), la régénération d'Acacia senegal peut se retarder pendant plusieurs mois (Sall, 1997). Pour de nombreuses essences agroforestières, un prétraitement spécial des graines est nécessaire pour obtenir une germination satisfaisante. Les prétraitements ne sont pas à l'origine de la germination des graines, mais les rendent capables de germer ultérieurement quand toutes les conditions requises sont réunies. C'est, par définition, les prétraitements réalisés avant, pendant ou après la conservation, qui permettent l'élimination de la dormance par leurs effets mécaniques, chimiques, physiologiques isolés ou associés (Debroux et al., 1998). C'est en fonction de la constitution de la coque des graines que le type de prétraitement est défini. Cependant, peu d'études ont porté jusqu'à présent sur le comportement germinatif des semences de cette essence en conditions contrôlées. C'est dans ce contexte et afin d'étudier l'influence de trois prétraitements sur la germination, qu'une expérience a été entreprise sur les graines d'Acacia senegal pour obtenir la meilleure germination de cette Légumineuse.

\section{Matériel et Méthodes \\ Provenance des graines}

Les semences utilisées dans le cadre de cette étude sont des graines collectées dans l'arrondissement de Moutourwa (département de Mayo-Kani) de septembre à décembre 2017 plus précisément à Laf $\left(14^{\circ} 10,800 \mathrm{E}\right.$, $10^{\circ} 12,000 \mathrm{~N}$ ). 


\section{Traitements prégerminatifs}

Des lots de semences d'Acacia senegal ont été soumis à trois traitements prégerminatifs avant d'être semés dans de substrat contenu dans des sachets de polyéthylène. Le prétraitement par l'acide sulfurique a consisté à tremper les graines d'Acacia senegal dans l'acide sulfurique concentré pendant neuf min puis à les retirer et les rincer à l'eau froide pendant 5 à 10 min pour éliminer les traces de l'acide. En ce qui concerne le prétraitement par ébouillantage, les graines saines d'A. senegal ont été trempées dans l'eau bouillante $\left(100^{\circ} \mathrm{C}\right)$ contenue dans un pot en terre cuite pendant trois min puis elles ont été rincées à deux reprises dans l'eau froide et trempées pendant 24 heures dans l'eau du robinet et sont conservées pour être semées le lendemain. Dans le cas de la scarification, cette technique a consisté à casser à l'aide d'une lame un petit bout du tégument de la graine sur le côté opposé à l'axe embryonnaire. Puis, ces traitements ont été comparés à des témoins non traités.

\section{Réalisation de semis et entretien}

Le semis a été effectué dans des sachets en polyéthylène de 18x10x8 $\mathrm{cm}^{3}$ qui contenaient un mélange de vertisols, de sable et de fumier (bouse de vache tamisée). Ce mélange de substrat a été stérilisé dans des bacs portés à $100^{\circ} \mathrm{C}$ pendant $60 \mathrm{mn}$ afin d'éliminer l'élément étranger susceptible de perturber l'expérimentation. Après remplissage des sachets, ceux-ci ont été disposés sous l'ombrage d'arbres composés d'Azadizachta indica. L'entretien a consisté à l'arrosage qui se passait deux fois par jour (matin et soir à $8 \mathrm{~h} 00$ et $17 \mathrm{~h} 00$ respectivement). Chaque fois que les mauvaises herbes apparaissaient, une opération de désherbage a été effectuée. Ceci a permis à la plante de mieux se développer. Quelques séances de pompage des insecticides ont été appliquées à chaque fois qu'il a été constaté l'apparition des attaques pour éliminer les insectes.

\section{Dispositif expérimental}

Après semis, les pots ont été disposés selon un dispositif en bloc aléatoire composé de quatre traitements par bloc. Trois répétitions par traitement ont été effectuées. Chaque unité expérimentale comptait 20 pots contenant chacun une graine. L'essai s'est déroulé du 29 mars 2018 au 21 avril 2018.

\section{Collecte des données}

A partir du 29 mars 2018, nous avons effectué tous les jours un comptage systématique des plantules ayant levé jusqu'au 21 avril 2018. La levée correspond à l'apparition d'une plantule avec deux feuilles cotylédonaires. L'atteinte de ce stade a également été prise en considération dans l'évaluation de la durée de la germination. Les paramètres suivants ont 
été déterminés : le délai d'attente (délai de germination) qui est le temps écoulé entre le semis et la première germination; la vitesse de germination (échelonnement) est le délai entre la première et la dernière germination; le taux de germination qui est obtenu à partir de la formule suivante: $\mathrm{T}=\mathrm{G} / \mathrm{N}$ avec $\mathrm{G}=$ nombre de graines germées et $\mathrm{N}=$ nombre de graines mises à germer par traitement.

\section{Analyse des données}

Les pourcentages, les moyennes et les erreurs standards des paramètres étudiés ont été calculés. La différence des valeurs notées est évaluée par une analyse de la variance (ANOVA) et par le test de Duncan pour le classement des moyennes.

\section{Résultats}

\section{Délai de germination}

Le délai de germination correspond au temps écoulé entre le semis et la première germination. Selon les résultats obtenus, on remarque que le délai de germination des graines varie suivant les traitements (Figure 2). La durée de germination des graines varie de 3 à 12 jours. A travers l'analyse des données de cette figure, on constate que les graines non traitées (témoins) ont une durée de germination la plus longue (12 jours). Par contre, elle est nettement plus courte au niveau des graines scarifiées soit 3 jours de germination. Pour les traitements à l'ébouillantage et l'acide sulfurique, le délai de germination est de 5 et 4 jours respectivement.

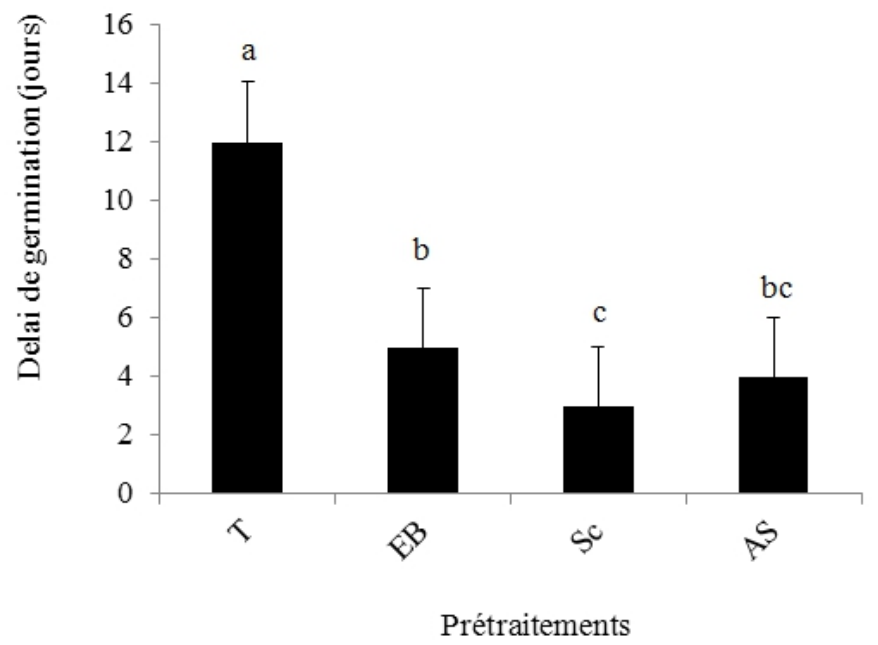

Figure 2 : Délai de germination $(\mathrm{T}=$ Témoin $; \mathrm{EB}=$ Eau bouillante $; \mathrm{Sc}=$ Scarification $; \mathrm{AS}=$ Acide sulfurique). Les histogrammes portant des mêmes lettres sont statistiquement identiques $(\mathrm{P}<0,05)$. 


\section{Taux de germination}

Le travail réalisé porte sur la recherche de l'influence des différents prétraitements sur le taux de germination des graines d'Acacia senegal. Pour le témoin, le taux de germination est égal à $26 \%$. Les taux de germination pour les prétraitements à l'acide sulfurique et à l'eau bouillante sont respectivement de $52 \%$ et $61 \%$. Tandis que, la germination atteint son maximum soit $87 \%$ pour les graines qui ont subi la scarification (Figure 3 ). Statistiquement l'analyse de la variance montre qu'il existe un effet très hautement significatif $(\mathrm{P}<0,001)$ des différents prétraitements sur le taux de germination.

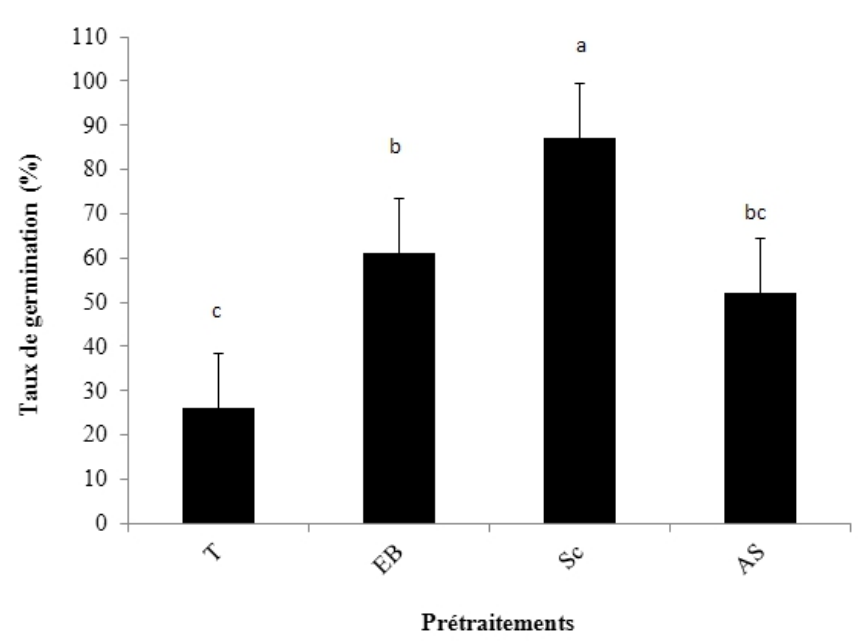

Figure 3 : Effet de prétraitements sur le taux de germination des graines d'Acacia senegal ; $\mathrm{T}=$ Témoin, $\mathrm{EB}=$ Eau bouillante $; \mathrm{Sc}=$ Scarification $; \mathrm{AS}=$ Acide sulfurique. Les histogrammes portant des mêmes lettres sont statistiquement identiques $(\mathrm{P}<0,05)$.

\section{Vitesse de germination}

La Figure 4 montre que $35 \%$ des graines ayant subi la scarification ont germé dès le $4^{\mathrm{e}}$ jour alors que chez celles ayant été traitées par l'acide sulfurique et l'eau bouillante, $16 \%$ et $17 \%$ ont germé respectivement. Par contre chez les graines témoin, seuls $8 \%$ ont germé après 13 jours. Au $21^{\text {ème }}$ jour, les taux de germination sont de $42 \%, 60 \%, 87 \%$ et $98 \%$ respectivement pour le témoin, le traitement à l'acide sulfurique, à l'eau chaude et pour la scarification. 


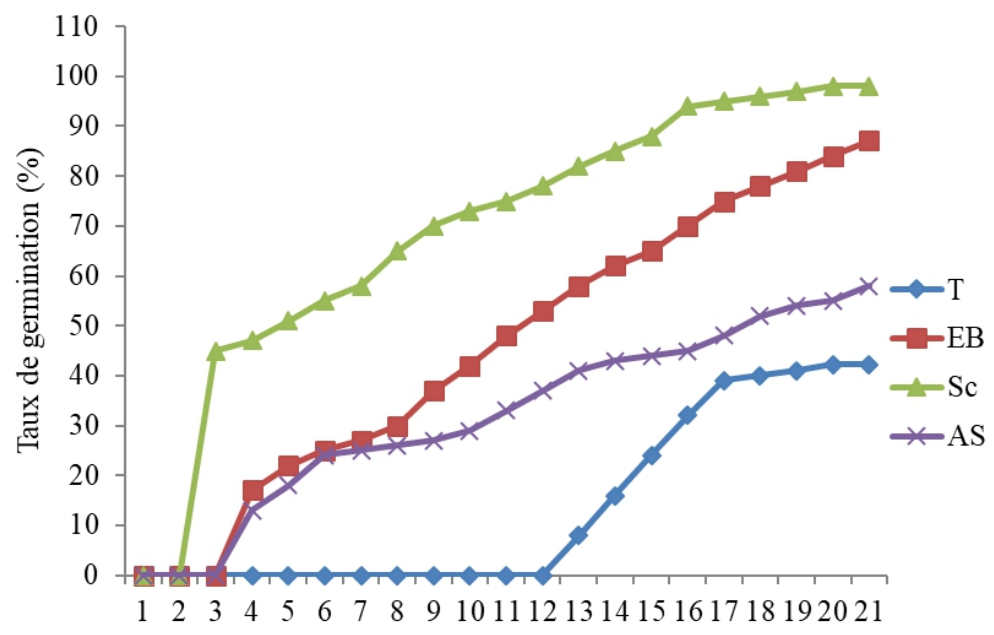

Temps (J)

Figure 4 : Effet des différents prétraitements sur la vitesse de germination des graines d'A. seneagl $(\mathrm{n}=3$ répétitions). $\mathrm{T}=$ Témoin $; \mathrm{EB}=\mathrm{Eau}$ bouillante $; \mathrm{Sc}=$ Scarification $; \mathrm{AS}=$ Acide sulfurique

\section{Discussion}

Cette étude a montré que pour avoir une germination rapide et homogène des graines d'Acacia senegal, le prétraitement des semences est nécessaire avant et après conservation de celles-ci. Les graines ayant subi des prétraitements avant leur semis ont des durées d'attente plus courtes que celles qui n'ont pas été traitées. En ce qui concerne le délai de germination, l'étude a également montré que les courts délais de germination sont obtenus avec les semences traitées à la scarification.

Pour les prétraitements des semences comparés dans le cadre de ce travail, les taux de germination les plus élevés sont obtenus avec la scarification et l'acide sulfurique. Par rapport à la scarification, ce traitement accélère la germination malheureusement il expose l'embryon et les cotylédons gorgés d'eau et de protéines à des pourritures et aux attaques parasitaires. C'est la raison pour laquelle toutes les graines n'ont pas germé à ce niveau. Le traitement à l'eau bouillante est un moyen efficace pour ramollir le tégument de la graine et pour réduire ainsi l'imperméabilité de celui-ci à l'eau (Aduradola \& Badru, 2004 ; Rolston, 1978 ; Tran \& Cavanagh, 1984). Malheureusement dans le cas de cette étude, le délai de germination a été un peu plus long que celui de la scarification. Cela s'explique par le fait que la coque de la graine n'a été suffisamment ramollie pour laisser passer l'eau et l'oxygène capable de déclencher les processus physiologiques. Ces assertions sont corroborent celles de Aduradola \& Badru (2004). Ahoton et al. (2009) expliquent cela par la durée de trempage et l'épaisseur des téguments. Selon 
ces auteurs, la durée de trempage des graines dans $1^{\prime}$ eau à $100^{\circ} \mathrm{C}$ dépend de l'épaisseur et de la dureté des téguments de la graine. En effet, l'utilisation de $1^{\prime}$ eau à $100^{\circ} \mathrm{C}$ dans le cadre de cette étude a permis non seulement de réduire le délai entre le semis et la première germination, la durée de germination des graines mais a également augmenté le pourcentage de germination.

Les taux de germination obtenus dans le cadre de ce travail sont comparables voire plus élevés que ceux observés par Nedjimi et al. (2014) par contre sont inférieurs à ceux observés par Ouédraogo (1986). En étudiant la germination des graines de Prosopis africana, l'auteur a trouvé un taux de germination variant entre 70 et $80 \%$ lorsque celles-ci sont traitées à l'acide sulfurique concentré pendant 60 à $90 \mathrm{mn}$ et trempées dans l'eau de robinet pour une durée de 24 heures. Des taux de germination plus ou moins semblables sont obtenus avec d'autres espèces dont les graines ont subi des prétraitements comparables aux nôtres: Faidherbia albida 95\% (Maydell, 1983; Prosopis julifora 80 à 90\% (Maydell, 1983; Canarium schweinfurthii $95 \%$ (Njoukam, 1997); Hyphaene thebaica 85 \% (Moussa et al. 1998). Les taux de germination obtenus dans le cas de notre étude montrent que nos traitements ont donné de résultats raisonnables.

Les principaux résultats obtenus dans le présent travail sur la germination des graines d'Acacia senegal montrent que la scarification a permis d'obtenir rapidement la germination que les autres prétraitements. En effet, les graines scarifiées ont germé le plus rapidement possible avec un taux de $45 \%$ dès le deuxième jour après le semis. Alors que celles traitées à l'eau bouillante et l'acide sulfurique ont non seulement de faibles taux de germination (17\% et $13 \%$ respectivement) mais leur délai de germination est aussi plus long (4 jours après semis). Ces résultats sont en ligne avec ceux obtenus par Bamba et al. (2018) qui ont enregistré les pourcentages les plus élevés chez Pterocarpus erinaceus pour les graines scarifiées. Les meilleurs taux de germination obtenus dans le cadre de travail s'expliquent par la facilité de l'embryon à germer et à émettre les racines au niveau des graines scarifiées alors dans les autres cas, la coque de la graine limiterait cette capacité à travers une dormance péricarpique (FAO, 1992). La dormance péricarpique a déjà été remarquée chez Pterocarpus angolensis (Laurie, 1974) et chez Pterocarpus santalinus (Naidu \& Rajendrudu, 2001). De plus, la scarification permet d'éliminer les graines non saines ou endommagés. Les prétraitements à l'eau bouillante et à l'acide sulfurique présentent de faibles taux de germination à cause de leur action négatif sur la graine. Dans le cadre de cette étude, à cause de ces autres prétraitements, certaines graines pourraient avoir été endommagées. Cette hypothèse a été émise par Agbogan et al. (2014) pour justifier le faible taux de germination constaté chez Haematostaphis barteri. 


\section{Conclusion}

Les résultats de cette étude ont montré que la scarification des graines à la lame de rasoir constitue la méthode la plus efficace permettant une germination rapide et homogène d'Acacia senegal. Cependant, le trempage à l'eau bouillante et le prétraitement à l'acide sulfurique ont des effets considérables sur la germination de cette espèce. Par ailleurs pour une meilleure politique de développement des espèces d'intérêt socioéconomique, la maîtrise de ses exigences de cette essence, toute en maintenant les conditions optimales pour sa germination en pépinière, est plus que nécessaire pour la création des parcs d'Acacia senegal dans la zone sahélienne du Cameroun.

\section{References:}

1. Abdou M. M., Alzouma Mayaki Z., Kadri A., Ambouta J.-M. K. \& Dan Lamso N., 2013. Effet de l'arbre Acacia senegal sur la fertilité des sols de gommeraies au Niger. Int. J. Biol. Chem. Sci. 7(6): 2328-2337.

2. Aduradola A. M. \& Badru, 2004, Aspects of germination in seeds of Afzelia Africana Sm. And Terminalia ivorensis A. Chev. Annales des Sciences Agronomiques du Bénin, 6(2) : 175-184.

3. Agbogan A., Bammite D., Tozo K. \& Akpagana K., 2014. Contribution à la multiplication, par graines et par bouturage de segments de tiges et de racines, de trois fruitiers spontanés de la région des savanes au Togo: Haematostaphis barteri Hook. F., Lannea microcarpa Engl. \& K. Krauss et Sclerocarya birrea (A. Rich.) Hochst. European Scientific Journal, 10 (6): 195- 211.

4. Ahoton L. E., Adjakpa J. B., M'po Ifonti M'po \& Akpo E. L., 2009. Effet des prétraitements des semences sur la germination de Prosopis africana (Guill., Perrot. et Rich.) Taub. (Césalpiniacées). Tropicultura, 27 (4): 233-238.

5. Anderson I. S. \& Sinclair F. L., 1993. Ecological Interactions in Agroforestry Systems. Agroforestry Systems 6(2): 58-61.

6. Bamba N., Ouattara N. D., Konan D., Bakayoko A. \& Tra Bi F. H., 2018. Effets de cinq prétraitements sur la germination du vène (Pterocarpus erinaceus Poir., Fabaceae) dans la Réserve du Haut Bandama (Côte d'Ivoire). European Scientific Journal, Vol.14, No.30 : 438-453.

7. Brenan J. P. M., 1983. Manuel sur la taxonomie des espèces d'acacias. Rome, Italie, Fao, 53 p.

8. CNUCED, 2016. Gomme arabique, un profil de produit de base par INFOCOMM Fonds de la CNUCED pour l'information sur les marchés des produits de base agricoles. 
9. Debroux L., Delvingt W., Mbolo M. \& Amougou A., 1998, La régénération du Moabi et du Mukulungu au Cameroun. Bois et forêts des tropiques, $255: 5-17$.

10. Diallo A., Agbangba E. C., Thiaw A. \& Guisse A., 2012. Structure des populations de Acacia senegal (L.) Willd dans la zone de Tessékéré (Ferlo nord), Sénégal. Journal of Applied Biosciences 59: 4366- 4374.

11. Dommergues Y. R., Duchoux E. \& Diem H. G., 1999. Les Arbres Fixateurs d'Azote : Caractéristiques Fondamentales et Rôle dans l'Aménagement des Ecosystèmes Méditerranéens et Tropicaux. CIRAD, Edition Espace, FAO, IRD: Montpellier.

12. Eisa M. A, Roth M. \& Sama G., 2008. Acacia Senegal (Gum Arabic Tree): Present Role and Need for Future Conservation/ Sudan: Proceeding "Competition for Resources in a Changing World: New Drive for Rural Development" Deutscher Tropentag, Hohenheim, pp. 1-5.

13. FAO, 1992. Guide de manipulation des semences forestières. Etude FAO forêt $20 / 2$ http://www.fao.org/docrep/006/AD232F/AD232F00.

14. Harmand J.-M., Njiti C. F. \& Peltier R., 1997. Restauration de la fertilité des sols par la jachère arborée. Cycle de l'azote, statut organique du sol, production des cultures. In : L'agroforesterie pour un développement rural durable. Recherche fondamentale et modélisation, applications tempérées et méditerranéennes. Atelier international, Montpellier, France, 23-29 juin 1997. Montpellier, France, Cirad, p. 135-142.

15. Ickowicz A., Friot D. \& Guérin H., 2005. Acacia senegal, arbre fourrager sahélien ? Bois et Forêts des Tropiques, 284 (2) : 59-69.

16. Jamal A. \& Huntsinger L., 1993. Deterioration of a sustainable agrosilvo-pastoral system in the Sudan: The gum gardens of Kordofan. Agroforestry Systems, 23 : 23-38.

17. Laurie M. V., 1974. Tree planting practices in African savannas. Food and Agriculture Organization of the United Nations, Rome. pp. 42-43.

18. Maydell V. H.-J., 1983, Arbres et arbustes du Sahel. Leurs caractéristiques et utilisations. Schriftenreihe der G.T.Z. (Deutsche Gesellschaft für Technische Zusammenarbeit), no 147, Eschborn, Allemagne.

19. Moussa H., Margolis H. A., Dube P. A. \& Odongo J., 1998, Factors affecting the germination of doum palm (Hyphaene thebaica Mart.) seeds from semi-arid zone of Niger, West Africa. Forest Ecology and Management, 104, 27-41. 
20. Naidu C. V. \& Rajendrudu G., 2001. Influence of kinetin and nitrogenous salts on seed germination of red sanders (Pterocarpus santalinus Linn. f.). Seed Science and Technology, 29 (3): 669-672.

21. Nedjimi B., Difi M. \& Haddioui A., 2014. Effets des différents prétraitements sur la germination des semences de pin d'Alep (Pinus halepensis Mill.). Revue des BioRessources 4 (2) : 40-45.

22. Njoukam R., 1997, Germination des semences et croissance de l'aielé Canarium schweinfurthii Engl. en plantation. In: Kapseu C. et Kayem G.J. (eds). Actes du 2ème séminaire international sur la valorisation du safoutier et autres oléagineux non conventionnels. Presses Universitaires de Yaoundé. Pp. 45-54.

23. Ouédraogo S. J., 1986, Sylviculture des essences locales: acquis de la recherche en la matière au Burkina Faso. IRBET/CTFT, 58 p.

24. Peltier R. \& Eyog-Matig O. 1988. Les essais d'agroforesterie au NordCameroun. Bois et forêts des tropiques, 217: 3-31 .

25. Peltier R., 1993. Les jachères à composante ligneuse. Caractérisation, conditions de productivité, gestion. In : La jachère en Afrique de l'Ouest. Atelier international, Montpellier, France, 2-5 décembre 1991. Montpellier, France, Orstom, coll. Colloques et séminaires, p. 67-88.

26. Rolston M. P., 1978, Water impermeable seed dormancy. Bot. Rev., 44: 365-396.

27. Saidou A. K., Hassane M., Abdoullatif Y. T. \& Karimou A., 2018. Effets d'Acacia senegal (L.) Willd. sur le Rendement du Niébé (Vigna unguiculata) au Niger, Afrique de l'ouest. European Scientific Journal, 14 (27) : 176-192.

28. Sall P. N., 1997: Le gommier et la gomme arabique. Note technique du projet RCS -sahel-1 507/RAF/43.

29. Tran V. N. \& Cavanagh A. K., 1984, Structural aspects of dormancy: In: D.R. Murray (ed.) Seed Physiology. V. II. Academic Press, Melbourne, pp. 1-44. 